\title{
Decitabine combined with CAG for the treatment of atypical chronic myeloid leukemia: a case report and literature review
}

\author{
Juan Cheng", Hao Zhang", Hai-Zhen Ma \\ Department of Hematology, The First Hospital of Lanzhou University, Lanzhou, China \\ \#These authors contributed equally to this work. \\ Correspondence to: Juan Cheng, MBBS, PhD. Professor of Medicine, Department of Hematology, The First Hospital of Lanzhou University, 1 \\ Donggangxilu Street, Lanzhou, China. Email: chenggu029@163.com.
}

\begin{abstract}
Atypical chronic myeloid leukemia BCR/ABL1 negative (aCML) is a rare hematopoietic stem/ progenitor cell disorder characterized by neutrophilia, high rate of transformation to acute myeloid leukemia and poor survival. Currently, there is no consensus on the treatment for aCML. In this study, we report the case of a 52-year-old female aCML patient treated with decitabine in combination with CAG chemotherapy who achieved complete remission after the first course. The patient was subsequently treated with three cycles of the same regimen as consolidation treatment. Approximately two months after the completion of the fourth cycle, the patient was in good general health with less than $0.01 \%$ minimal residual leukemic cells. The findings of this case report indicate that decitabine in combination with CAG chemotherapy may be an effective treatment for aCML.
\end{abstract}

Keywords: Decitabine; CAG chemotherapy; atypical chronic myeloid leukemia (aCML); treatment; case report

Submitted Sep 08, 2019. Accepted for publication Jun 18, 2020.

doi: $10.21037 /$ tcr-19-1806

View this article at: http://dx.doi.org/10.21037/tcr-19-1806

\section{Introduction}

Atypical chronic myeloid leukemia, BCR-ABL1 negative (aCML) represents a rare disorder of hematopoietic stem/ progenitor cells that exhibits clinical and morphologic similarity to chronic myeloid leukemia (CML) but lacks both the Philadelphia chromosome and BCR-ABL1 fusion gene. Based on the myelodysplastic and myeloproliferative characteristics, aCML is classified as a myelodysplastic/ myeloproliferative neoplasm (MDS/MPN) along with chronic myelomonocytic leukemia (CMML), juvenile myelomonocytic leukemia (JMML), and myelodysplastic syndrome/myeloproliferative neoplasm, unclassifiable (MDS/MPN, U) according to the World Health Organization (WHO) classification system (1). More specifically, aCML is characterized by leukocytosis (WBC count $\geq 13 \times 10^{9} / \mathrm{L}$ ) due to increased numbers of neutrophils and their precursors, prominent dysgranulopoiesis, and a differentiated blood count showing $>10 \%$ neutrophil precursors (promyelocytes, myelocytes, metamyelocytes), $<2 \%$ basophils, $<10 \%$ monocytes and $<20 \%$ blasts in the blood and bone marrow (2).

aCML is associated with a poor prognosis and a high risk of transformation to acute myeloid leukemia. No standard of care exists for the treatment of aCML, and until now, allogeneic hematopoietic stem cell transplantation (alloHSCT) has been the only curative treatment option (3). Other treatment strategies include hypomethylating agents (decitabine or azacitidine), novel agents based on targetable mutations (e.g., JAK inhibitor for JAK2 or CSF3R mutation; MEK inhibitor for RAS mutation), second-line options (e.g., PEG IFN-a) and supportive (e.g., erythropoiesisstimulating agents). Here, we report a patient with aCML who was successfully treated with decitabine in combination with the CAG regimen (low-dose cytarabine, aclarubicin and G-CSF). We present the following case in accordance with the CARE Reporting Checklist.

\section{Case presentation}

A 52-year-old female was hospitalized at the First Hospital 


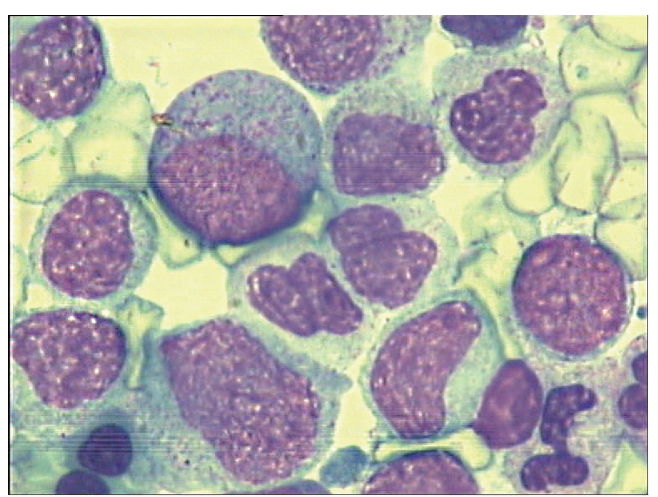

Figure 1 Morphological examination of the bone marrow showing active bone marrow hyperplasia of atypical chronic myeloid leukemia (×100 magnification). Obviously identifiable marrow blasts, a relatively high proportion granulocytes with unbalanced development. Wright's stain.

of Lanzhou University with increasing fatigue and an intermittent cough that had persisted for 1 week. The patient had a normal medical history with no known drug allergies, no travel to epidemic areas and no known exposure to toxins. There was no family history of malignancy. On physical examination, some petechiae and edema were identified on both lower limbs. No involvement of the superficial lymph nodes or rales was identified. The cardiac rhythm was regular, without murmurs. In addition, the patient's abdomen was soft and the liver was not palpable. No splenic tenderness or costovertebral angle tenderness was observed.

A routine blood test and peripheral blood smear revealed a WBC count of $34.81 \times 10^{9} / \mathrm{L}$, with a differential of $77.0 \%$ neutrophils, $7.0 \%$ myeloblasts, $8.0 \%$ neutrophilic myelocytes, $5.0 \%$ lymphocytes, $1 \%$ eosinophils and $2.0 \%$ monocytes, and a hemoglobin concentration and platelet count of $120 \mathrm{~g} / \mathrm{L}$ and $32 \times 10^{9} / \mathrm{L}$, respectively.

Significant granulocytic hyperplasia was observed upon morphological examination of the bone marrow, with $16.0 \%$ myeloblasts, $37 \%$ myelocytes and meta myelocytes observed in the total nucleated marrow cell count (Figure 1).

Flow cytometry revealed that $81.5 \%$ of the total nucleated cells were myeloblasts and myeloid cells, with $7.0 \%$ myeloblasts showing prominent expression of CD33, CD34, HLA-DR, CD7, CD13, CD38, CD56, CD117 and MPO and partial expression of CD123 with abundant dysplasia. Cytogenetic analysis demonstrated a normal chromosome karyotype, 46, XX [11]. Molecular genetic analysis by PCR indicated that BCR/ABL fusion genes,
Janus kinase 2 (JAK2) V617F, MPL W515L/K, and CALR mutations were negative. The patient was also negative for the rearrangements of PDGFR $\alpha$, and PDGFR $\beta$. However, detection of CEBPA gene mutations by whole exome sequencing demonstrated two mutations of CEBPA were detected in the bone marrow sample (c.273_274insC Figure 2A; c.959_960insGGTGCTGGTGCTGGAGCT GACCAGTGA; Figure 2B) Based on these findings, aCML was diagnosed.

The patient's 4 siblings were not completed HLAmatches and she refused incompatible allogeneic transplantation. She received induction treatment consisting of decitabine at $20 \mathrm{mg} / \mathrm{d}$ for 5 days and G-CSF $300 \mu \mathrm{g} / \mathrm{d}$ for priming (the dose of G-CSF was adjusted to the amount of blood routine), in combination with aclarubicin at $10 \mathrm{mg} / \mathrm{d}$ for 4 days, and cytarbine at $10 \mathrm{mg} / \mathrm{m}^{2} \mathrm{q} 12 \mathrm{~h}$ for 14 days. During induction therapy, the patient experienced infection and hemorrhage caused by myelosuppression, which disappeared after symptomatic treatment.

After the first cycle, the WBC count decreased to $8.3 \times 10^{9} / \mathrm{L}$, and the platelet count improved to $544 \times 10^{9} / \mathrm{L}$. One month after initial treatment, a second morphological examination of the bone marrow was performed, revealing that the bone marrow contained $2 \%$ myeloblasts and $17 \%$ myelocytes, with no granulocytic hyperplasia or dysplasia. The patient received a second cycle of decitabine in combination with CAG as consolidation therapy for aCML. Three months after initial treatment, a third morphological examination of the bone marrow was performed, which revealed that the bone marrow contained $1.5 \%$ myeloblasts, with no granulocytic hyperplasia or dysplasia. Subsequently, she received two more cycles of decitabine in combination with CAG chemotherapy. A routine blood test revealed a WBC count of $5.31 \times 10^{9} / \mathrm{L}$, and a hemoglobin concentration and platelet count of $125 \mathrm{~g} / \mathrm{L}$ and $165 \times 10^{9} / \mathrm{L}$. A bone marrow examination two months after completion of the last cycle revealed no myeloblasts and minimal residual leukemic cells less than $0.01 \%$ detected by flow cytometry. The CEBPA mutation was negative. At present, nine months after initial treatment, the patient continues to undergo further clinical observations and follow-up (Figure 3).

Written informed consent was obtained from the patient for publication of this manuscript and any accompanying images.

\section{Discussion}

Compared to CML, which is characterized by the consistent 
A

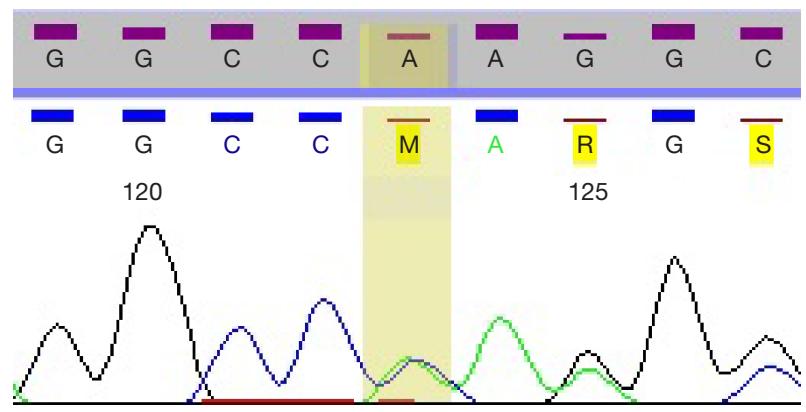

B
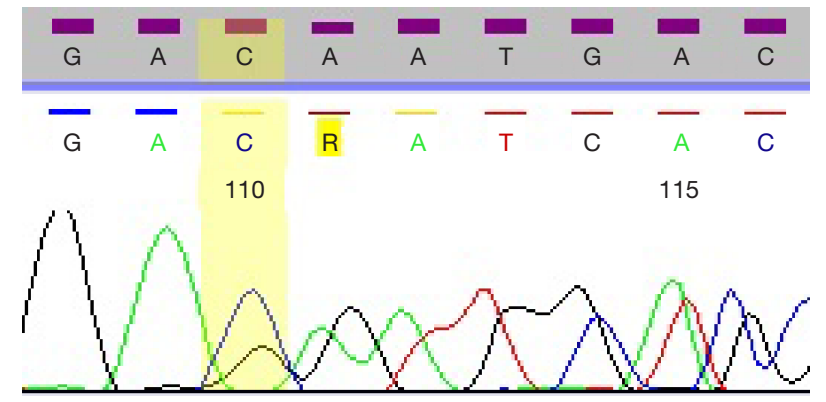

Figure 2 The second generation sequence analysis of CEBPA.

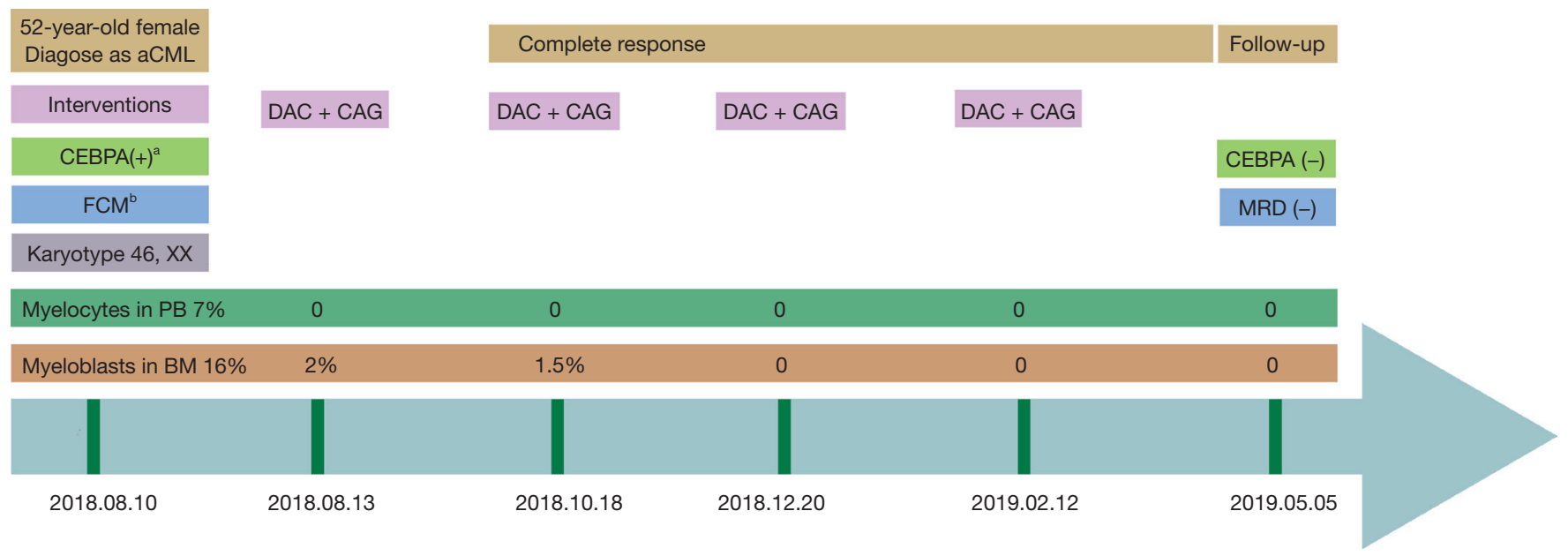

Figure 3 Case report timeline. a: positivity for CEBPA, negativity for JAK2 V617F, MPL W515L/K, CALR, PDGFR $\alpha$ and PDGFR $\beta$. b:CD33, CD34, HLA-DR, CD7, CD13, CD38, CD56, CD117 and MPO and partial expression of CD123 with abundant dysplasia. FCM, flow cytometry. MRD, minimal residual disease. DAC, decitabine. Interventions: decitabine at $20 \mathrm{mg} / \mathrm{d}$ for 5 days and G-CSF $300 \mu \mathrm{g} / \mathrm{d}$ for priming (the dose of G-CSF was adjusted to the amount of blood routine), in combination with aclarubicin at $10 \mathrm{mg} / \mathrm{d}$ for 4 days, and cytarbine at $10 \mathrm{mg} / \mathrm{m}^{2} \mathrm{q} 12 \mathrm{~h}$ for 14 days.

presence of the Philadelphia chromosome, no specific karyotypic abnormalities characterize aCML. Chromosomal changes include single or double abnormalities or complex karyotype aberrations, where chromosomes $7,8,12,13$, $14,17,19,20$ and 21 are frequently involved. $-7 / 7 \mathrm{q}-,+8$, $20 \mathrm{q}-$, or $117 \mathrm{q}$ are commonly reported and are detectable in other myeloid neoplasms (2). Similarly, the genetic mutations in aCML are nonspecific, SETBP1 (25-33\%), RAS (10-35\%), ASXL1 (66\%), SRSF2 (40\%), and TET2 $(29 \%)$ mutations are frequently reported in aCML $(3,4)$, whereas CBL (8-10\%), CSF3R (3\%), JAK2 (3-8\%) Kit $(1-4 \%)$, FLT3 $(3 \%)$ are infrequently reported $(4,5)$. The patient we reported here presented with a normal karyotype and CEBPA double mutations, which are usually reported in AML and associated with good prognosis. The CEBPA gene, located on 19q13.11, encodes CCAAT/enhancerbinding protein-alpha $(\mathrm{CEBP} \alpha)$, which affects transcription and DNA damage responses. CEBPA gene mutations have been reported in $4 \%$ of aCML, $4 \%$ of MDS/MPN-U, and 4-20\% of CMML cases and are associated with unfavorable prognosis (4). However, a report indicated that CEBPA double mutations may be associated with good prognosis in aCML as it is in AML (5).

Previous studies have shown that the median overall survival (OS) of aCML was 12.4 to 29 months (5), and $25 \%$ to $40 \%$ of aCML transformed to acute myeloid leukemia (6). HSCT is the only therapeutic option with curative intent for aCML. However, only a few studies have 
evaluated allogeneic HSCT in aCML patients. Koldehoff et al. (7) demonstrated the outcome of $9 \mathrm{aCML}$ patients who received allogeneic HSCT. All patients treated with allogeneic HSCT were alive at the time of analysis with a median follow-up of 55 months after transplant. Only 1 patient treated with syngeneic HSCT relapsed 19 months posttransplant, and this patient was successfully retransplanted with cells from the original donor. Mittal et al. (8) described unfavorable outcomes of 7 aCML patients who received allogeneic bone marrow $(n=5)$ or peripheral blood stem cell $(n=2)$ transplantation. Five patients died because of aGVHD $(n=1)$, cGVHD $(n=1)$, sepsis $(n=1)$, disease $(n=1)$ and an unknown cause $(n=1)$ after a follow-up of 3-26 months. A recent study described allo-HSCT outcomes of 42 aCML patients in which 26 patients achieved complete remission, with 51\% 5-year OS, 36\% 5-year RFS, and 24\% non-relapse mortality (NRM). However, transplant-related mortality and relapse rates were $24 \%$ and $40 \%$ at 5 years, respectively (9). It is currently unknown whether HSCT can modify the adverse prognosis related to gene mutations in the context of aCML.

Hydroxyurea or IFN may help control hyperleukocytosis and clinical symptoms, and a few patients achieved transient partial or complete remission with median survival ranging from 14 to 25 months. These options are only suitable for older or frail patients who cannot tolerate other therapies (4,5,7-10). Emerging agents include JAK2 inhibitors, CSF3R inhibitors, and MEK inhibitors, which are targeted therapies that may lead to favorable response based on genetic analysis (11-13). As of now, however, the application of these new therapies in aCML patients is limited to preclinical studies or clinical trials.

Decitabine is a hypomethylating agent that shows a promising and safe clinical response in MDS and CMML. A limited number of studies have evaluated the activity and safety of decitabine in patients with aCML. In the case series of Kantarjian et al. (14), 7 patients with Ph-negative CML received decitabine at $50-75 \mathrm{mg} / \mathrm{m}^{2}$ every 12 hours for five days and 4 patients achieved objective responses with severe myelosuppression. Tong et al. (15) reported more favorable outcomes in a study of $4 \mathrm{aCML}$ patients who were treated with decitabine $20 \mathrm{mg} / \mathrm{m}^{2}$ daily for 5 days, with 3 patients achieving CR after one cycle. One patient did not achieve complete remission after two cycles of decitabine. Hausmann et al. (16) first reported a 49-year-old Caucasian aCML patient complicated with paraneoplastic large-vessel vasculitis who achieved complete hematologic response after four cycles of decitabine $\left(20 \mathrm{mg} / \mathrm{m}^{2}\right.$ for 5 days $)$ and accepted allo-HSCT after the completion of six cycles. The patient died on day +49 because of aGVHD. Jiang et al. (17) demonstrated that single-agent decitabine was successfully used in two patients with aCML. Both patients were treated with four cycles of decitabine $\left(20 \mathrm{mg} / \mathrm{m}^{2}\right.$ for 5 days $)$ and achieved CR. Based on the presence of significant granulocytic dysplasia and hyperplasia, we decided to treat this patient with decitabine, aclarubicin and low-dose cytarabine. She achieved a complete hematologic response after one cycle and underwent further clinical observations and follow-up. We thought that this treatment shows a good early and safe response, However, multiple courses of such treatment could cause severe myelosuppression and sometimes fatal complications, such as severe infections. In order to prevent and deal with the limitations of this situation. In this case, after the chemotherapy, the patient was admitted to a laminar flow ward, injected with stimulating factors, infused blood products, and used antibiotics. The effect of treatment is satisfactory.

\section{Conclusions}

Despite the previously discussed results, there remains no standard treatment for aCML. The role of HSCT in aCML is not well established, however, it is the only therapeutic option with curative intent that is currently available. Our case report reveals that the CEBPA double mutations may also be associated with good prognosis in aCML. The use of decitabine in combination with CAG chemotherapy can be effective for the treatment of aCML patients who are ineligible for HSCT.

\section{Acknowledgments}

Funding: None.

\section{Footnote}

Conflicts of Interest: All authors have completed the ICMJE uniform disclosure form (available at http://dx.doi. org/10.21037/tcr-19-1806). The authors have no conflicts of interest to declare.

Ethical Statement: The authors are accountable for all aspects of the work in ensuring that questions related to the accuracy or integrity of any part of the work are appropriately investigated and resolved. All procedures 
performed in studies involving human participants were in accordance with the Declaration of Helsinki (as revised in 2013). The parent and guardians have given their written informed consent to publish the case (including publication of images).

Open Access Statement: This is an Open Access article distributed in accordance with the Creative Commons Attribution-NonCommercial-NoDerivs 4.0 International License (CC BY-NC-ND 4.0), which permits the noncommercial replication and distribution of the article with the strict proviso that no changes or edits are made and the original work is properly cited (including links to both the formal publication through the relevant DOI and the license). See: https://creativecommons.org/licenses/by-nc-nd/4.0/.

\section{References}

1. Arber DA, Orazi A, Hasserjian R, et al. The 2016 revision to the World Health Organization classification of myeloid neoplasms and acute leukemia. Blood 2016;127:2391-405.

2. Patnaik MM, Barraco D. Targeted next generation sequencing and identification of risk factors in World Health Organization defined atypical chronic myeloid leukemia. 2017;92:542-8.

3. Piazza R, Valletta S, Winkelmann N, et al. Recurrent SETBP1 mutations in atypical chronic myeloid leukemia. Nat Genet 2013;45:18-24.

4. Zoi K, Cross NC. Molecular pathogenesis of atypical CML, CMML and MDS/MPN-unclassifiable. Int J Hematol 2015;101:229-42.

5. Gotlib J. How I treat atypical chronic myeloid leukemia. Blood 2017;129:838-45.

6. Wang SA, Hasserjian RP, Fox PS, et al. Atypical chronic myeloid leukemia is clinically distinct from unclassifiable myelodysplastic/myeloproliferative neoplasms. Blood 2014;123:2645-51.

7. Koldehoff M, Beelen DW, Trenschel R, et al. Outcome of hematopoietic stem cell transplantation in patients with atypical chronic myeloid leukemia. Bone Marrow Transplant 2004;34:1047-50.

Cite this article as: Cheng J, Zhang H, Ma HZ. Decitabine combined with CAG for the treatment of atypical chronic myeloid leukemia: a case report and literature review. Transl Cancer Res 2020;9(8):5015-5019. doi: 10.21037/tcr-19-1806
8. Mittal P, Saliba RM, Giralt SA, et al. Allogeneic transplantation: a therapeutic option for myelofibrosis, chronic myelomonocytic leukemia and Philadelphianegative/BCR-ABL-negative chronic myelogenous leukemia. Bone Marrow Transplant 2004;33:1005-9.

9. Onida F, de Wreede LC, van Biezen A, et al. Allogeneic stem cell transplantation in patients with atypical chronic myeloid leukaemia: a retrospective study from the Chronic Malignancies Working Party of the European Society for Blood and Marrow Transplantation. Br J Haematol 2017;177:759-65.

10. Dao KH, Tyner JW. What's different about atypical CML and chronic neutrophilic leukemia? Hematology Am Soc Hematol Educ Program 2015;2015:264-71.

11. Shanavas M, Popat U, Michaelis LC, et al. Outcomes of Allogeneic Hematopoietic Cell Transplantation in Patients with Myelofibrosis with Prior Exposure to Janus Kinase 1/2 Inhibitors. Biol Blood Marrow Transplant 2016;22:432-40.

12. Borthakur G, Popplewell L, Boyiadzis M, et al. Activity of the oral mitogen-activated protein kinase kinase inhibitor trametinib in RAS-mutant relapsed or refractory myeloid malignancies. Cancer 2016;122:1871-9.

13. Khanna V, Pierce ST, Dao KH, et al. Durable Disease Control with MEK Inhibition in a Patient with NRASmutated Atypical Chronic Myeloid Leukemia. Cureus 2015;7:e414.

14. Kantarjian HM, O'Brien S, Cortes J, et al. Results of decitabine (5-aza-2'deoxycytidine) therapy in 130 patients with chronic myelogenous leukemia. Cancer 2003;98:522-8.

15. Tong X, Li J, Zhou Z, et al. Efficacy and side-effects of decitabine in treatment of atypical chronic myeloid leukemia. Leuk Lymphoma 2015;56:1911-3.

16. Hausmann H, Bhatt VR, Yuan J, et al. Activity of singleagent decitabine in atypical chronic myeloid leukemia. J Oncol Pharm Pract 2016;22:790-4.

17. Jiang $\mathrm{H}, \mathrm{Wu} \mathrm{Z}$, Ren LI, et al. Decitabine for the treatment of atypical chronic myeloid leukemia: A report of two cases. Oncol Lett 2016;11:689-92. 